\title{
AFS dynamics in a short-lived active region
}

\author{
F. Zuccarello ${ }^{1}$, V. Battiato ${ }^{1}$, L. Contarino ${ }^{2}$, P. Romano ${ }^{1}$, D. Spadaro ${ }^{2}$, and L. Vlahos ${ }^{3}$ \\ 1 Dipartimento di Fisica e Astronomia - Sezione Astrofisica, Università di Catania, via S. Sofia 78, 95125 Catania, Italy \\ e-mail: fzu@ct.astro.it \\ 2 INAF - Osservatorio Astrofisico di Catania, via S. Sofia 78, 95125 Catania, Italy \\ 3 Department of Physics, University of Thessaloniki, 54124 Thessaloniki, Greece
}

Received 4 April 2005 / Accepted 5 July 2005

\section{ABSTRACT}

In the framework of the study on active region emergence, we report the results obtained from the analysis of the short-lived (7 days) active region NOAA 10407. The data used were acquired during an observational campaign carried out with the THEMIS telescope in IPM mode in July 2003, coordinated with other ground- and space-based instruments (INAF-OACT, DOT, BBSO, MDI/SOHO, EIT/SOHO, TRACE). We determined the morphological and magnetic evolution of NOAA 10407, as well as the velocity fields associated with its magnetic structures. Within the limits imposed by the spatial and temporal resolution of the images analyzed, the first evidence of the active region formation is initially observed in the transition region and lower corona, and later on (i.e. after about $7 \mathrm{~h}$ ) in the inner layers, as found in a previous analysis concerning a long-lived, recurrent active region. The results also indicate that the AFS formed in the active region shows typical upward motion at the AFS's tops and downward motion at the footpoints. The velocity values relevant to the upward motions decrease over the evolution of the region, similarly to the case of the recurrent active region, while we notice an increasing trend in the downflow velocity during the early phases of the time interval analyzed by THEMIS. On the other hand, the AFS preceding legs show a higher downflow than the following ones, a result in contrast with that found in the long-lived active region. The chromospheric area overhanging the sunspot umbra shows an upward motion of $\sim 2 \mathrm{~km} \mathrm{~s}^{-1}$, while that above the pores shows a downward motion of $\sim 4 \mathrm{~km} \mathrm{~s}^{-1}$.

Key words. Sun: activity - sunspots - Sun: magnetic fields

\section{Introduction}

It is now well established that the appearance of active regions on the Sun is due to the emergence of magnetic flux tubes from subphotospheric layers. More precisely, observations seem to indicate that active regions form due to the emergence of several small (radius $\sim 200 \mathrm{~km}$ ), intense $\left(500 \mathrm{G}, \sim 10^{18} \mathrm{Mx}\right.$ ) flux tubes, which are separate during the first phase of their appearance, but that soon cluster to $2-4 \times 10^{3} \mathrm{G}$ over $100 \mathrm{~km}$ (Fragos et al. 2004, and references therein). The flux tubes clustering tendency takes place until new magnetic flux emerges from subphotospheric layers and vanishes as soon as this emergence ceases (Zwaan 1985).

From a more specific phenomenological point of view, the first signature of emerging flux in the photosphere is a granular dark lane, with faculae or pores forming near the edges of this lane and strong (1-2 $\left.\mathrm{km} \mathrm{s}^{-1}\right)$ downdrafts near pores. The rise velocity of emerging flux tubes in photosphere is estimated to be $\sim 1-2 \mathrm{~km} \mathrm{~s}^{-1}$. The first signature of magnetic flux emergence in the chromosphere are $\mathrm{H}_{\alpha}$ brightenings or bright plages and $\mathrm{H} \alpha$ surges (Kurokawa \& Kawai 1993) while in the following hours a bundle of dark arches crossing the polarity inversion line, called arch filament system (AFS), appears (Bruzek 1968). The AFS arches show a rising velocity at their tops of $10-15 \mathrm{~km} \mathrm{~s}^{-1}$ and downflows at their endpoints of $10-50 \mathrm{~km} \mathrm{~s}^{-1}$, have lengths of $1-3 \times 10^{4} \mathrm{~km}$ and a lifetime of the order of $\sim 20$ min (Shibata et al. 1989, and references therein; Yoshimura \& Kurokawa 1999).

However, despite the detailed knowledge we have today on the formation and emergence of active regions (see, e.g., van Driel-Gesztelyi 2002), it is not yet possible to forecast whether newly emerging magnetic flux tubes will give rise to a fully developed active region, or whether they will disappear within a few hours/days. A contribution to the solution of this question has been given by high angular and temporal resolution observations carried out at the THEMIS telescope, aimed at studying the details of the very initial phases of active region formation.

Spadaro et al. (2004, Paper I), using data acquired during an observational campaign carried out at the THEMIS telescope in IPM mode, coordinated with other ground- and space-based instruments, analyzed the first evolutionary phases of a recurrent active region (NOAA 10050). The main result concerned the dynamic evolution of the arch filament system: the line of sight velocities determined from Doppler measurements, while confirming that the loops forming the AFS show an upward motion at their tops and a downward motion at their extremities, 


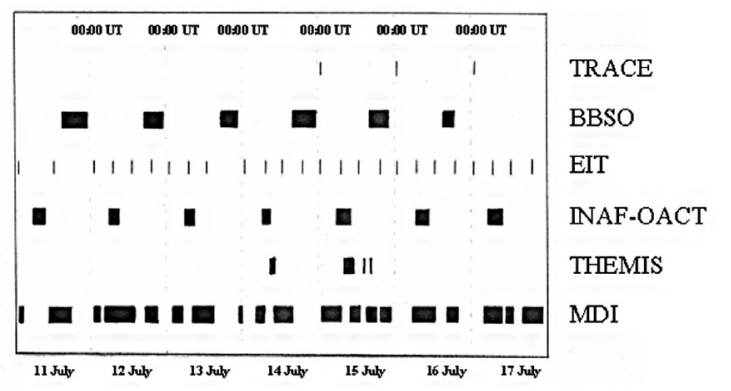

Fig. 1. Data coverage for the different instruments from July 11 to July 17, 2003.

also indicated that the upward motion decreases as the active region evolves. Moreover, some asymmetries between the preceding and the following sides of NOAA 10050 were shown: the $\mathrm{p}$-side was larger than the f-side and moved westward much faster than the f-side receded eastward; the AFS f-side exhibited higher downflows than the p-side. It was also found that the first signs of the active region formation were seen in the transition region and lower corona and later on (i.e. after about $6 \mathrm{~h}$ ) in the lower atmospheric layers. Moreover, the initial increase in the magnetic flux was synchronous with the active region appearance in the transition region and lower corona, and the rate of increase of the magnetic flux was steeper during the first 6 days. These results were interpreted as due to the presence of a mechanism decelerating the magnetic flux emergence as more flux tubes rise towards higher atmospheric layers.

To determine whether these characteristics are shared by all emerging active regions, or whether some elements can be used to discriminate between active regions having a full evolution and others decaying after a short time, we have carried out a similar study on a short-lived active region, NOAA 10407 , characterized by a lifetime of 7 days, observed during a subsequent Campaign performed with the THEMIS telescope (July 2003). The study was aimed: to determine the morphology and dynamics of magnetic elements (AFS arches, pores) during their emergence and development, to determine their characteristic lifetime, topology and associated velocity fields and to compare them with the results obtained for the long lived active region NOAA 10050 studied in Paper I.

\section{Observations}

\subsection{Data description}

We have studied Active Region NOAA 10407, that appeared on July 11 and vanished on July 17, 2003, using data acquired by several instruments (THEMIS/IPM, INAF-OACT, DOT, BBSO $\mathrm{H} \alpha$ telescope, EIT/SOHO, MDI/SOHO, TRACE). Figure 1 shows the data coverage for each instrument used in the campaign. The active region was observed by THEMIS on July 14 and 15 .

The images obtained by the Telescope Heliographique pour l'Etude du Magnetisme et des Instabilites Solaires (THEMIS) (Arnaud et al. 1998) operating in IPM mode (Cavallini 1998) were acquired in 18 spectral points, 12 along the profile of the H $\alpha$ line $(\lambda=6562.92 \AA,+/-0.25 \AA,+/-0.50 \AA,+/-0.75 \AA$,

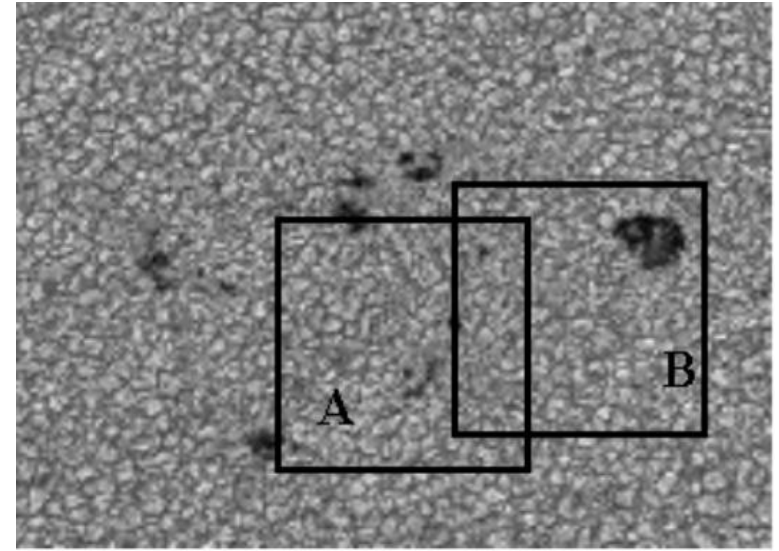

(a)

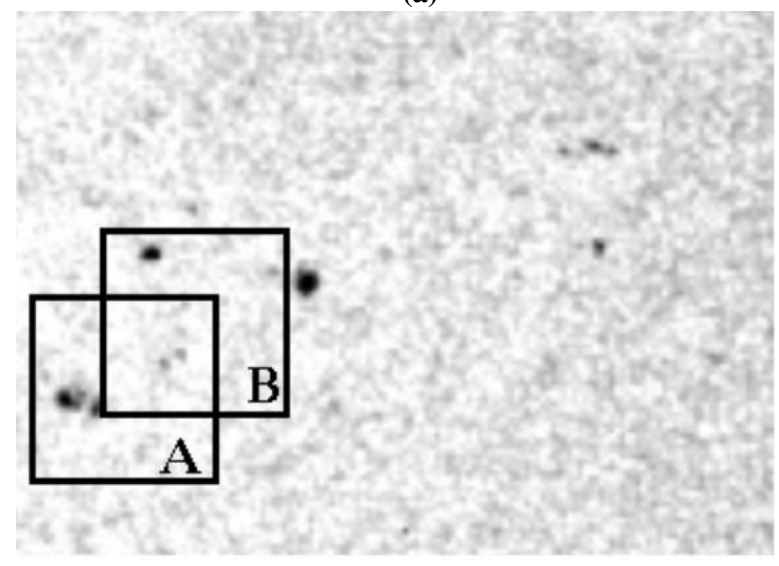

(b)

Fig. 2. a) Image of NOAA 10407 acquired by DOT in the red continuum at $6350 \AA$ on July 14. The field of view (fov) is $\sim 53000 \times 40000 \mathrm{~km}^{2}$. b) WL image of the active region acquired by TRACE on July 15 , with two of the three fields of view scanned by THEMIS. The fov is $\sim 100000 \times 70000 \mathrm{~km}^{2}$. The squares $A$ and $B$ indicate the two fields of view scanned during the THEMIS run. In these as well as the other images North is on the top and West on the right.

+/-1.00 $\AA,+/-1.50 \AA,-13.3 \AA$, with an exposure time of $300 \mathrm{~ms})$ and 6 along the Fe I line $(\lambda=5576.012 \AA,+/-0.040 \AA$, $+/-0.060 \AA,-0.090 \AA$, with an exposure time of $500 \mathrm{~ms})$. At the same time images in broad band centered at $5380.960 \AA$ were acquired. The period employed to acquire the images in the 18 spectral points is $\sim 50 \mathrm{~s}$. The temporal resolution between two images is $\sim 3 \mathrm{~s}$ and the spatial resolution is $\sim 0.4 \operatorname{arcsec}$ (see also Paper I).

On July 14 we acquired images of NOAA 10407 using the THEMIS telescope in two different fields of view (hereinafter called sectors A and B, see the squares in Fig. 2a), which were almost aligned along the East-West direction and overlapped by $\sim 10$ arcsec. The sequences were carried out in such a manner that, after a complete scan along both $\mathrm{H} \alpha$ and FeI lines was performed in the field of view A, the telescope was pointed toward the field of view B, then back to A, and so on, so that the two fields of view were alternatively scanned. The following day, three fields of view were scanned: the first two were approximately equal to sectors A and B previously scanned (see Fig. 2b), while the third field of view considered a filament lying on the East side of the active region (this field of view is 
Table 1. THEMIS data set.

\begin{tabular}{ccccc}
\hline \hline Day & Heliographic & Observing time (UT) & Selected fields of view & Performed sequences \\
& coordinates & & & \\
\hline July 14, 2003 & $10 \mathrm{~N}$ 02W & $8: 32-9: 51$ & 2 & 75 \\
July 15, 2003 & $10 \mathrm{~N} 17 \mathrm{~W}$ & $8: 19-10: 10$ & 3 & 105 \\
\hline
\end{tabular}

outside the TRACE image shown in Fig. 2b). The THEMIS dataset is reported in Table 1 . In the same table, the heliographic coordinates of NOAA 10407 are shown in Col. 2.

During the observing campaign full disc images were acquired at INAF-Catania Astrophysical Observatory with a cadence of $15 \mathrm{~min}$ in the centre of the $\mathrm{H} \alpha$ line $(6563 \AA)$ and in its red wing $(6568 \AA$ ) with a pass band of $0.25 \AA$ (Zuccarello et al. 2002), in order to choose the target observed through the restricted field of view of THEMIS and to monitor the photospheric and chromospheric configuration of the selected active region. During shorter time intervals the photospheric configuration could also be monitored by means of the Dutch Open Telescope (DOT) (Rutten 1999) and TRACE WL images.

In order to study the magnetic field configuration and evolution of NOAA 10407, we used full disc magnetograms acquired by MDI/SOHO (Scherrer et al. 1995) from 19:14 UT on July 10 to $17: 14$ UT on July 18, with an average time cadence of $96 \mathrm{~min}$ and a spatial resolution of 4 arcsec. To determine the details of the magnetic configuration, we also used high resolution magnetograms acquired by MDI on July 14 and 15 .

Synoptic full disc images at $171 \AA, 195 \AA, 284 \AA$ and $304 \AA$ were acquired by EIT/SOHO (Delaboudinière et al. 1995) each day at 1:00, 7:00, 13:00 and 19:00 UT, while TRACE (Handy et al. 1999) acquired one image of the active region per day at $171 \AA$ on July 15-17 (1:30 UT), and one per day at $1600 \AA ̊$ on July 16 (1:30 UT) and 17 (2:30 UT).

\subsection{NOAA 10407 global morphology}

On July 11, no sign of NOAA 10407 was visible in the photospheric images acquired at INAF-Catania Astrophysical Observatory during the time interval 6:00-14:00 UT. The appearance of the region in the photosphere was recorded by MDI only at 16:00 UT. All the WL images show that the morphology of the region does not exhibit significant variations from July 12 to July 17: only one sunspot and some pores are visible (see Fig. 2). On July 15 and 16, NOAA 10407 is already a small decaying region with some pores, while on July 17, it appears as a tiny pore.

The appearance of NOAA 10407 in $\mathrm{H} \alpha$ was first recorded by the Big Bear Solar Observatory on July 11 at 15:48 UT. The region, observed at INAF-Catania Astrophysical Observatory since 6:32 UT on July 12, shows a compact facular structure. In BBSO images acquired on the same day at 15:59 UT an AFS is observed between the two pairs of pores, as well as a filament in the Southern part of the active region. On July 14 the formation of a small filament on the South-East of the spot is recorded (see the arrow in Fig. 3a). On July 15, a new external

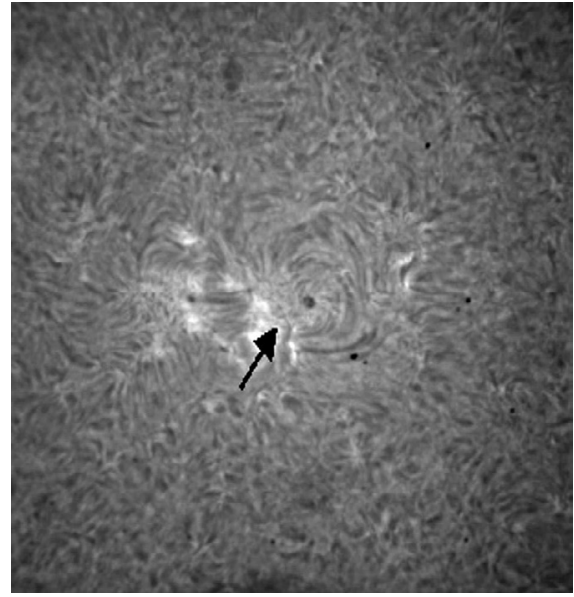

(a)

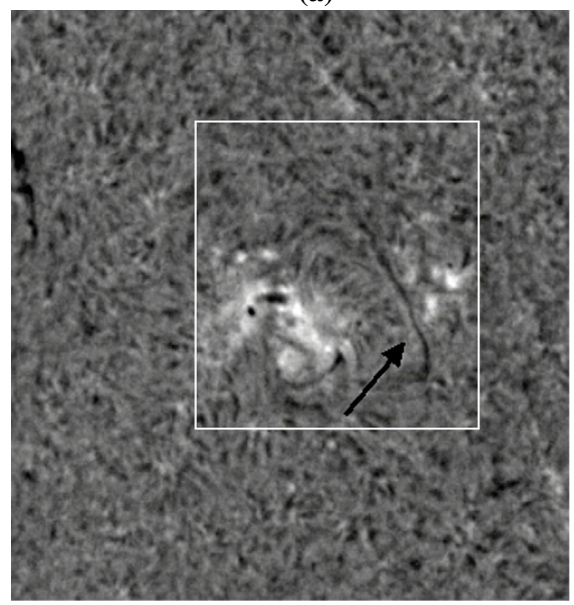

(b)

Fig. 3. BBSO $\mathrm{H} \alpha$ images of NOAA 10407 acquired on July 14 at 18:02 UT a) and 15 at 17:59 UT b). The fov is $\sim 210000 \times$ $210000 \mathrm{~km}^{2}$. The white square in image b) indicates the TRACE fov relevant to the same day (see Fig. 4a).

filament in the Western part of the active region is observed (see the arrow in Fig. 3b).

The active region initially appeared in the images acquired by EIT/SOHO in the $195 \AA$ band on July 11 at 8:48 UT as a small, bright structure, while it became visible in the images acquired in the other EIT bands in the following hours. In the subsequent days, the active region scarcely evolved and some loop systems appeared. In particular, on July 15 and 16, a loop system is visible in the Eastern part of the fov (see Fig. 4, showing TRACE $171 \AA$ images). Comparing BBSO and TRACE images acquired on July 15 (i.e. Figs. $3 b$ with $4 a$ ), it is possible to infer the co-spatiality of the chromospheric AFS with the inner loops of the bright loop system on the East of the TRACE 


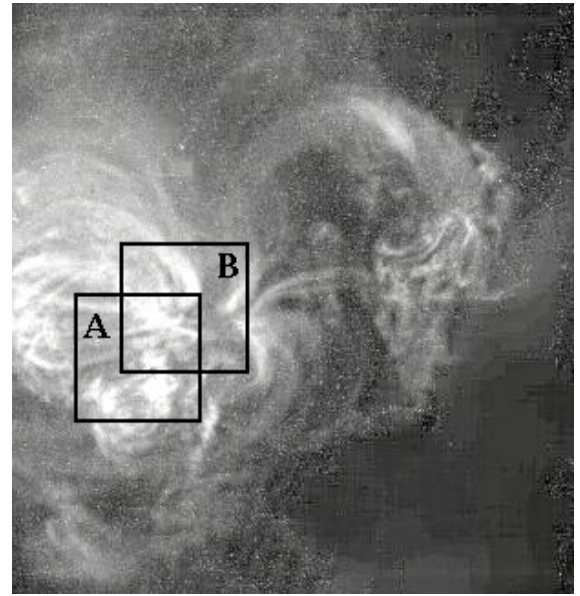

(a)

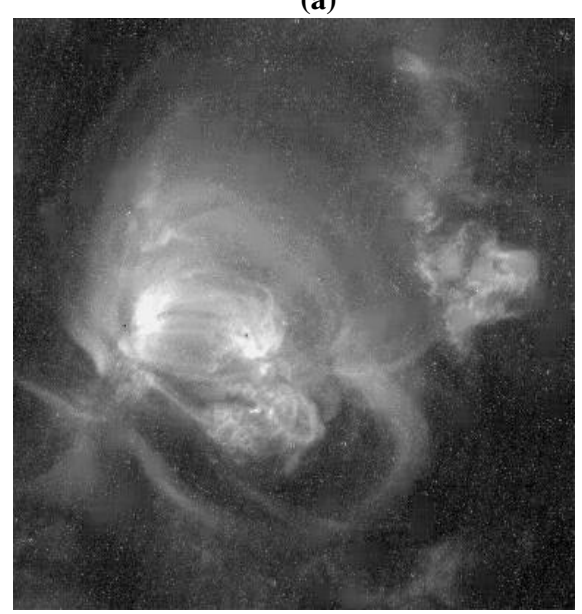

(b)

Fig. 4. $171 \AA$ images acquired by TRACE at 1:30 UT on July 15 a) and at 1:30 UT on July $16 \mathbf{b}$ ). The fov is $\sim 130000 \times 130000 \mathrm{~km}^{2}$. The squares A and B in image a), relevant to July 15, indicate the two fields of view scanned by THEMIS in this day.

fov as well as the co-spatiality between the bright faculae at the AFS footpoints and the edges of the outer coronal loops (see also Yoshimura \& Kurokawa 1999, for the temporal and spatial relation between AF arches and coronol loops).

We can summarize the results obtained from the analysis of these observations as follows: the active region NOAA 10407 is first observed to appear in the upper atmosphere (EIT $195 \AA$ image) on July 11 at 8:48 UT, while the first record of the relevant sunspot group is at 16:00 UT (MDI image), with heliographic coordinates N09, E29. The active region scarcely develops, exhibiting the formation of a single spot, several pores and some filaments. It is observed on the solar disc only for 7 days, before completely decaying, and during its lifetime it does not show any flare activity.

\section{Data analysis}

\section{1. $M D I$}

To study the rate of emergence of magnetic flux in NOAA 10407, we selected one MDI full disc magnetogram every $96 \mathrm{~min}$, from 19:14 UT on July 10 to 17:14 UT on July 18.
For each magnetogram we limited our analysis to a subfield of $111 \times 87$ pixels $\left(155000 \times 122000 \mathrm{~km}^{2}\right)$, centered on the active region and we determined both the positive and negative emerging magnetic flux, taking into account the corrections required by the projection effects.

To determine the magnetic field configuration of the active region at higher atmospheric levels, we used an extrapolation code based on a method introduced by Alissandrakis (1981), who proposed a solution for the boundary value problem of a force-free magnetic field with constant $\alpha$ by using Fourier Transforms. The model is based on the assumptions that the magnetic field is force-free not only in the corona, but also in the lower levels and that it vanishes at infinity. Because of its linearity, the force-free magnetic field equation:

$$
\nabla \times \boldsymbol{B}=\alpha \boldsymbol{B}
$$

can be numerically solved using Fourier Transforms. The code takes as an input the value of the normal component of the magnetic field at the boundary (in our case the boundary is the photosphere and the normal component to the boundary is the longitudinal magnetic field deduced by MDI high resolution magnetograms). Using this method, the code reconstructs the 3-D magnetic field above the photosphere, giving as an output three arrays with the values of the magnetic field components in the considered space.

\subsection{THEMIS/IPM}

We corrected the THEMIS/IPM data applying the standard dark current and flat field corrections (e.g., Contarino et al. 2003), as described in Paper I.

We then reconstructed the profiles of FeI and $\mathrm{H} \alpha$ lines in each spatial pixel by fitting the corresponding signals obtained by the monochromatic images with a linear background and a Gaussian shaped line, obtaining the values of the fit parameters by the maximum likelihood method. The Gaussian line fitting allows determination of the position of the line centroid within a fraction of the wavelength resolution obtained by the IPM instrument for the two spectral lines considered (see also Spadaro et al. 2000).

The values of velocity along the line of sight (los) in photosphere (through FeI line) and chromosphere (through $\mathrm{H} \alpha$ line) have been deduced from the Doppler shift of the centroid of the line profile in each spatial point with respect to the median of the line centroid in the whole field of view, as in Paper I. The median is used as wavelength reference, since there is no absolute wavelength reference in IPM. We estimated the uncertainty affecting the velocity measurements in a statistical way, considering the standard deviation of the centroid of the FeI and $\mathrm{H} \alpha$ line profiles determined in all points of the whole field of view with respect to the relevant median. The so-estimated errors in the velocity are $\pm 0.2 \mathrm{~km} \mathrm{~s}^{-1}$ and $\pm 1 \mathrm{~km} \mathrm{~s}^{-1}$ for the Fe I and $\mathrm{H} \alpha$ line, respectively.

In order to determine the horizontal photospheric velocity field we applied the cross correlation procedure, developed by Kitay et al. (1997), to broad band images observed at different times. We refer the reader to Paper I for a detailed description of the adopted method. 


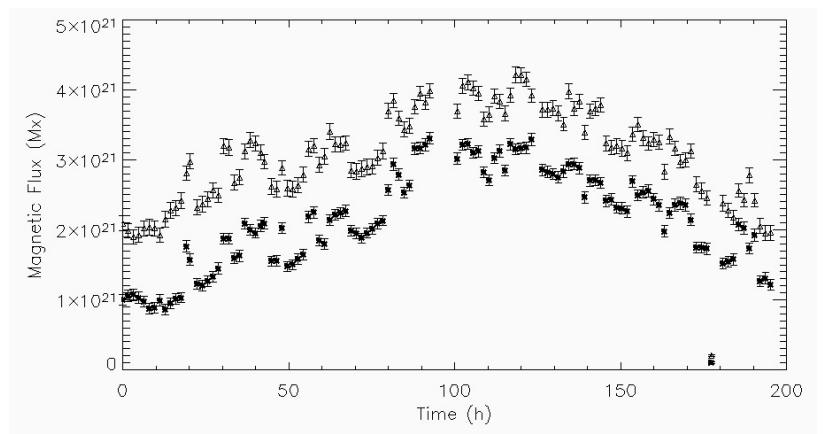

Fig. 5. Magnetic flux in NOAA 10407 from 19:14 UT on July 10 to 17:14 UT on July 18, as deduced every 96 min from MDI full disc magnetograms. The asterisks and the triangles represent the positive and negative magnetic flux (in absolute value), respectively. The bars indicate the estimated errors.

\section{Results}

\subsection{Magnetic flux and field configuration}

Figure 5 reports both the positive (asterisks) and negative (triangles) magnetic flux, in absolute value, as a function of time. The origin in abscissa corresponds to 19:14 UT, on July 10. During the first $10 \mathrm{~h}$ we do not see any significant variation of the magnetic flux, which exhibits an average value of $1.0 \times 10^{21} \mathrm{Mx}$ and $2.0 \times 10^{21} \mathrm{Mx}$ for the positive and negative polarity, respectively. However, approximately $13 \mathrm{~h}$ after the beginning of the observing run, we notice an increase in the magnetic flux. This time roughly corresponds to the appearence of the active region in the EIT/SOHO images at $195 \AA$. At about $120 \mathrm{~h}$ after the beginning we register a total increase by $\sim 1.9 \times 10^{21} \mathrm{Mx}$ and $\sim 1.5 \times 10^{21} \mathrm{Mx}$ in the positive and negative magnetic flux, respectively, even if their behaviour vs. time is not monotonic, but almost oscillating. Afterwards, we notice a smoother continuous decrease in the magnetic flux.

The longitudinal component of the magnetic field in NOAA 10407 on July 14 and 15 is shown in Fig. 6, and the magnetic field extrapolation for the two days is reported in Fig. 7. Both extrapolations show several magnetic field lines connecting the pores on the East of the single sunspot present in the active region. The field lines closer to the photosphere clearly exhibit the same length $(\sim 15000 \mathrm{~km})$ and orientation of the AFS arches visible in the $\mathrm{H}_{\alpha}$ images (see Figs. 3, 9 and 10) and discussed later on. The field lines at higher levels satisfactorily represent the loop systems visible in the TRACE $171 \AA$ images (Fig. 4). Moreover, these extrapolations also suggest that the single sunspot is connected to magnetic elements apparently not belonging to NOAA 10407, and located westward.

Using MDI magnetograms with a time cadence of $24 \mathrm{~h}$, acquired from July 12 to the day when the active region vanished (17 July), we determined the centre of symmetry of each polarity for each day. We used an IDL procedure that allows us to select the magnetic flux distribution for each polarity over a certain selected area and successively determines its centre of symmetry. Considering the displacements of the centres of symmetry between two consecutive days and dividing by the corresponding time interval we deduced their velocities (see the arrows in Fig. 8). We found that during the first phase of

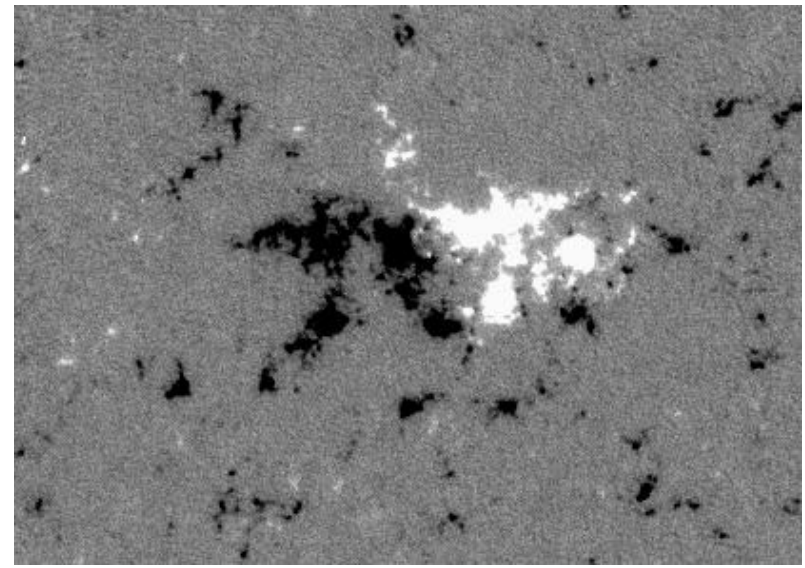

(a)

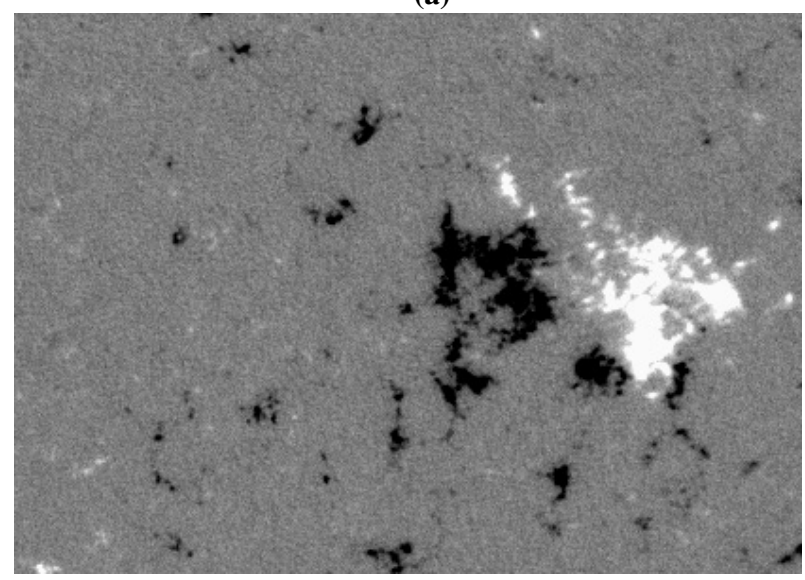

(b)

Fig. 6. High resolution magnetograms of NOAA 10407 acquired by MDI at 10:34 UT on July 14 a) and at 6:08 UT on July 15 b). The fov is $\sim 176000 \times 126000 \mathrm{~km}^{2}$.

the active region lifetime the following (negative) polarity is characterized by a motion directed toward the inversion line, instead of moving away from it. We determined an average velocity of $\sim 0.07 \mathrm{~km} \mathrm{~s}^{-1}$ and of $\sim 0.06 \mathrm{~km} \mathrm{~s}^{-1}$ for the negative and positive polarities, respectively.

\subsection{AFS morphology and evolution}

Figures 9 and 10 report the images of sectors A and B obtained from the observations carried out with THEMIS on July 14 and 15 , respectively. The images acquired in the centre of the $\mathrm{H} \alpha$ line clearly show the arch filament system located between the two emerging magnetic polarities of the Eastern pores. On July 14 this AFS is composed of $\sim 10$ arch filaments (AF), approximately parallel and located across the polarity inversion line (PIL). They have several lengths, up to a maximum of $\sim 15000 \mathrm{~km}$, and appear to outline the extrapolated magnetic field lines connecting the opposite polarities of the two pores, as reported in Fig. 7a.

We can distinguish 5 AFs in Fig. 9c: 2, named $\beta$ and $\gamma$, seeming almost stable during the time interval 9:00-9:35 UT, and 3, named $\alpha, \epsilon$ and $\delta$ more dynamic; in particular, the formation of AF $\delta$ occurs in the time interval between 9:17 and 9:24 UT and later this AF seem to merge with AF $\beta$. In 


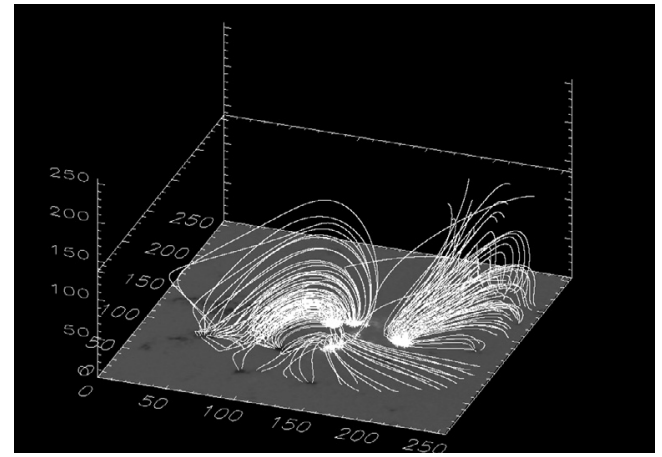

(a)

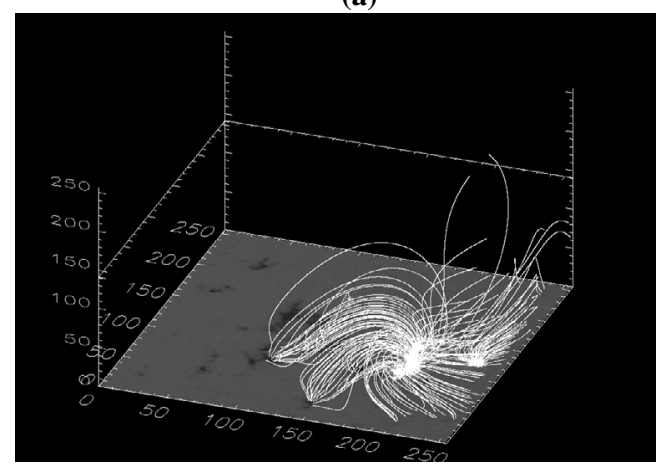

(b)

Fig. 7. Magnetic field extrapolations in NOAA 10407, superposed on the relevant MDI magnetograms, for July 14 a) and 15 b).

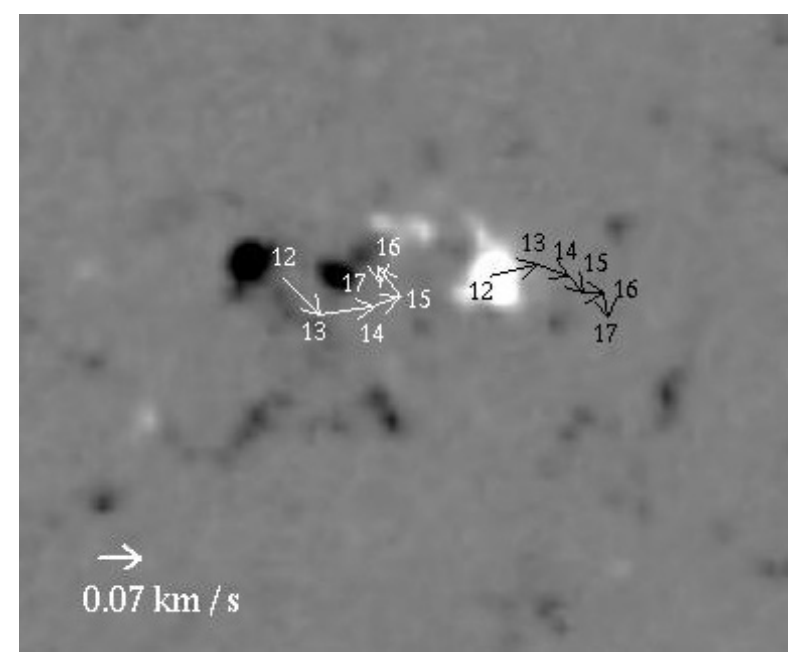

Fig. 8. MDI magnetogram acquired at 4:46 UT on July 12. The arrows indicate the velocities of the polarity centres of symmetry in the following days, till July 17 at 4:46 UT. The numbers at the arrow edges indicate the days considered for the determination of the centres of symmetry. The length of the arrows is referred to the white arrow at the left bottom, indicating a velocity of $0.07 \mathrm{~km} \mathrm{~s}^{-1}$.

the photosphere, observed in the line of Fe I (5576.012 $\AA$ ) at 9:19 UT (see Fig. 9a), this area is characterized by some pores. Figure $9 \mathrm{~b}$ shows Sector B, where a small sunspot $(d \sim$ $6000 \mathrm{~km}$ ) is visible in the North-West area.

Moreover, in both A and B sectors, due to their partial overlapping, it is possible to distinguish a small filament (length $\sim 14000 \mathrm{~km}$ ), located between the South-West pores and the sunspot, and characterized by a very dynamic evolution (see the arrows in Figs. 9c and 9d). We postpone the discussion on the evolution of this structure to a future paper, focusing here on the behaviour of the AFS.

On July 15, when the magnetic flux reaches its maximum value (see Fig. 5), we still observe the AFS in sector A, in the area between the two groups of pores (Fig. 10c), but with characteristics different from those observed the day before: the AFs are still parallel, but more numerous, thinner, closer to each other, with smaller dimensions and a faster dynamic evolution. We could distinguish 6 AFs: 3 (named $\kappa, \mu$ and $\tau$ ) seeming rather stable and 3 (named $\eta, \chi$ and $\omega$ ) more dynamic. There are also some small arch filaments which appear in one scan only, hence with a lifetime shorter than $2 \mathrm{~min}$, even if we cannot rule out that their disappearance is due to changes in the seeing conditions. They are not indicated in Fig. 10c.

In sector B we can see that the area overlying the pores is brighter than the surrounding regions; moreover, the small filament observed the day before is not visible in these images.

\subsection{Velocity fields}

Figure 11 shows the los velocity maps obtained from the analysis of the $\mathrm{H} \alpha$ profile in sectors A and B on July 14 at 9:22 and 9:23 UT, respectively. We plot the velocity contours every $1 \mathrm{~km} \mathrm{~s}^{-1}$ over the image taken in the centre of the $\mathrm{H} \alpha$ line. Thick and thin contours indicate positive (upward) and negative (downward) los plasma motion, respectively. The velocity map relevant to sector A indicates that the top of each AF is characterized by a plasma positive velocity, which indicates the upward motion of the entire AF structure, rising in the chromosphere. At the same time, the ends of the AFs generally exhibit negative velocities, which can be interpreted as signatures of a downward plasma motion along the magnetic field lines at the footpoints of each AF. We refer the reader to Paper I for a critical assessment of the properties of the motions in the AFS as deduced from the los plasma velocities study.

Table 2 reports the maximum los upward velocity at the top of the AFs, as well as the maximum downward velocities at their ends, determined on July 14 . We note the highest value of $\sim 4 \mathrm{~km} \mathrm{~s}^{-1}$ at the top of AF $\beta$, while the preceding end of $\mathrm{AF} \beta$ is characterized by the highest downward los velocity $\left(\sim-12 \mathrm{~km} \mathrm{~s}^{-1}\right)$. Moreover, both AFs $\gamma$ and $\delta$ show an asymmetry in the los velocities at their ends: there is a stronger downflow in the preceding footpoints than in the following ones. A similar comparison is not possible in AFs $\alpha$ and $\beta$ because their following footpoints are outside the selected field of view. Table 3 reports the corresponding velocity values determined on July 15 . We note a significant reduction in both the rising velocity of the top and the downward velocities at the ends of the AFs, by a factor of about 2 . However, the asymmetry in the los velocities at the ends is still evident.

In Fig. 12 we report the plots of the maximum upflow velocities determined at the top of AFs $\beta$ (diamonds) and $\gamma$ (asterisks) during 4 sequences carried out on July 14 (8:49-9:00, 9:00-9:12, 9:12-9:22, 9:22-9:35 UT). These plots indicate that in both arches there is a slow decrease in the upflow velocity 


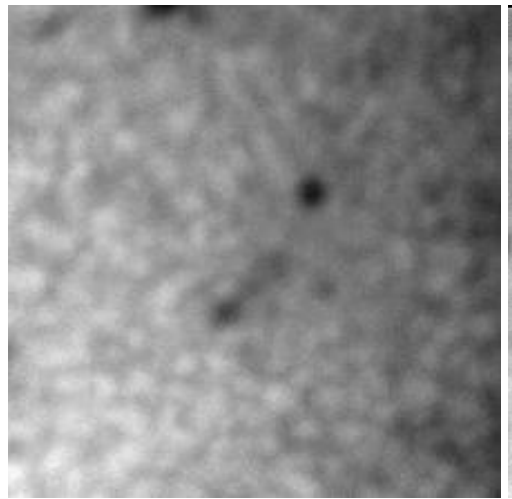

(a)

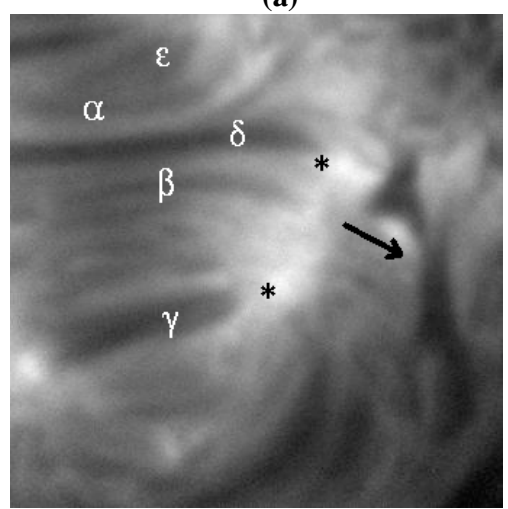

(c)

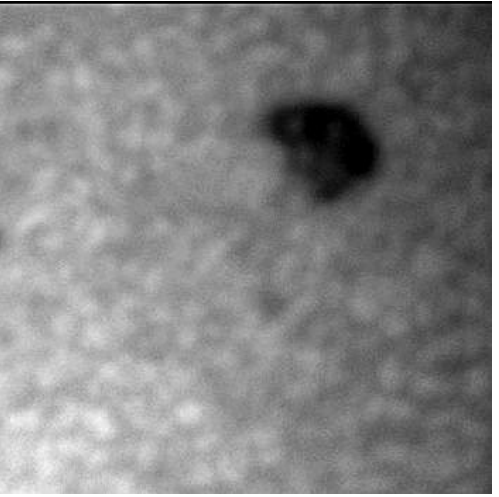

(b)

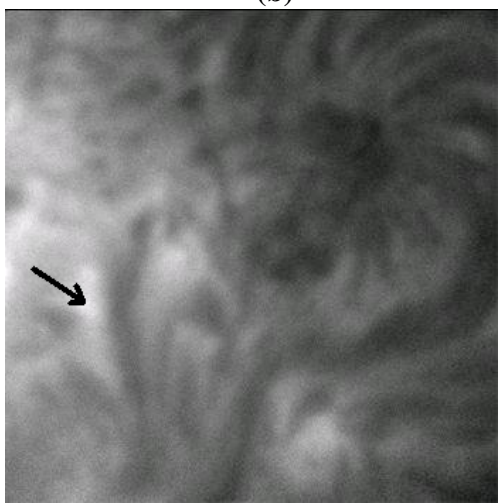

(d)

Fig. 9. Photospheric images of Sector A a) and Sector B b) and chromospheric images of the same fields of view c) and d) acquired by THEMIS on July 14. The letters indicate the AFS arches analyzed. The asterisks in c) indicate the position of the pores visible in a).

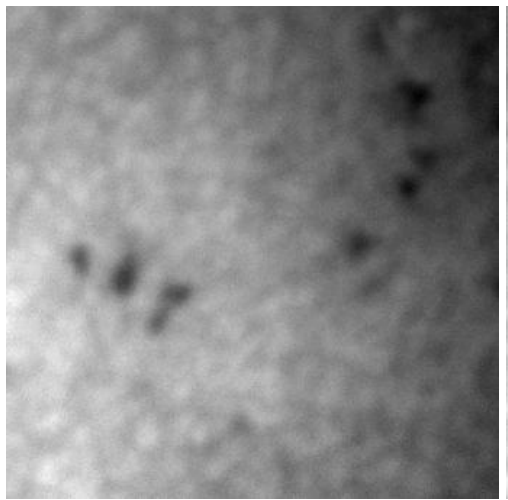

(a)

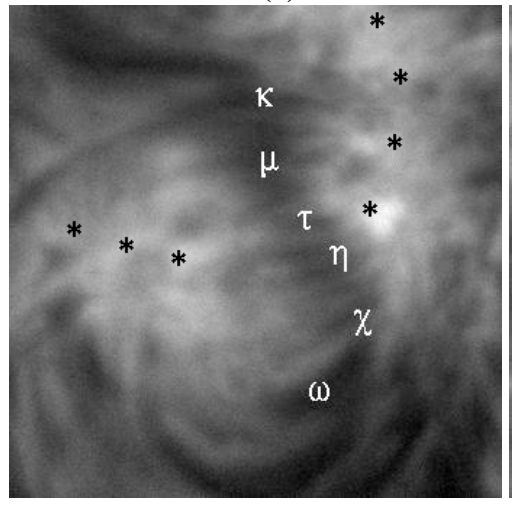

(c)

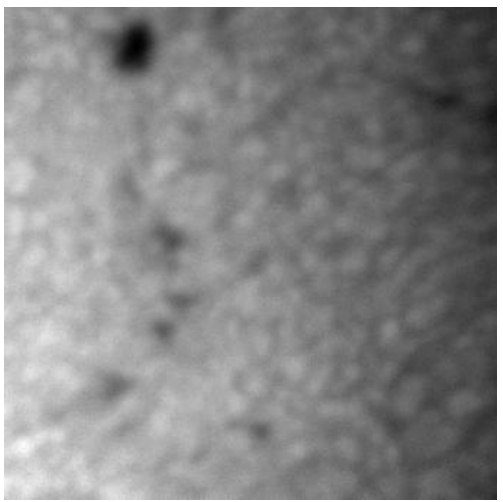

(b)

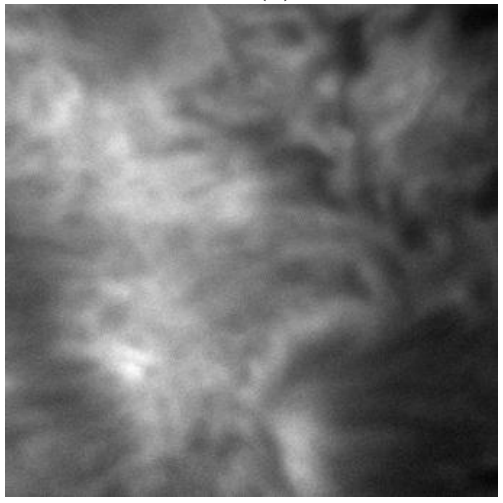

(d)

Fig. 10. As in Fig. 9 for the images acquired by THEMIS on July 15. 


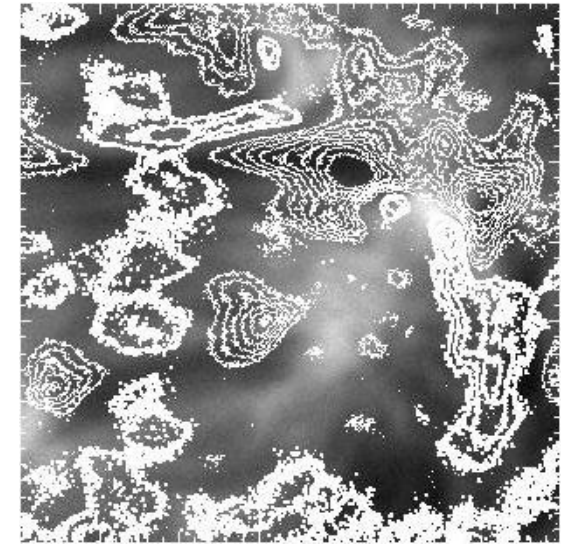

(a)

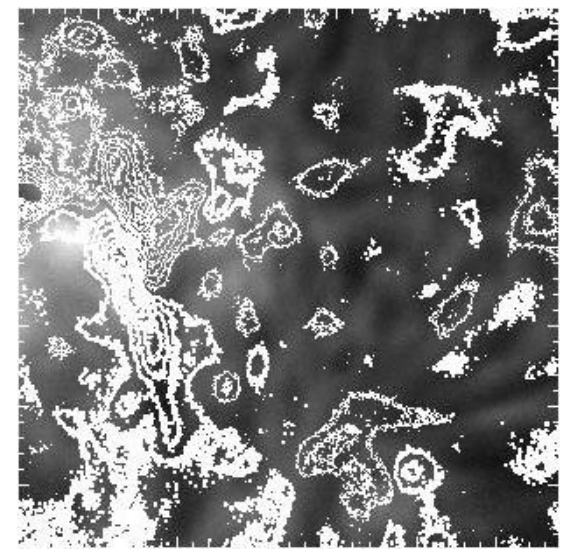

(b)

Fig. 11. Velocity maps obtained using the $\mathrm{H} \alpha$ profile in sector A a) and sector B b) on July 14, at 9:22 and 9:23 UT, respectively. Each map is plotted over an image acquired in the centre of the $\mathrm{H} \alpha$ line. Thick contours indicate upward plasma motions, while thin contours indicate downward plasma motions. The velocity contours are drawn every $1 \mathrm{~km} \mathrm{~s}^{-1}$.

Table 2. Maximum velocity at the top and ends of the AFs observed on July 14.

\begin{tabular}{lccc}
\hline \hline AF & $\begin{array}{c}\text { Velocity } \\
\text { of the top }\left(\mathrm{km} \mathrm{s}^{-1}\right)\end{array}$ & $\begin{array}{c}\text { Velocity of the } \\
\text { following end }\left(\mathrm{km} \mathrm{s}^{-1}\right)\end{array}$ & $\begin{array}{c}\text { Velocity of the } \\
\text { preceding end }\left(\mathrm{km} \mathrm{s}^{-1}\right)\end{array}$ \\
\hline$\alpha$ & +2 & - & -5 \\
$\beta$ & +4 & - & -12 \\
$\gamma$ & +2 & -3 & -6 \\
$\delta$ & +2 & -1 & -7 \\
\hline
\end{tabular}

Table 3. Maximum velocity at the top and ends of the AFs observed on July 15 .

\begin{tabular}{cccc}
\hline \hline AF & $\begin{array}{c}\text { Velocity } \\
\text { of the top }\left(\mathrm{km} \mathrm{s}^{-1}\right)\end{array}$ & $\begin{array}{c}\text { Velocity of the } \\
\text { following end }\left(\mathrm{km} \mathrm{s}^{-1}\right)\end{array}$ & $\begin{array}{c}\text { Velocity of the } \\
\text { preceding end }\left(\mathrm{km} \mathrm{s}^{-1}\right)\end{array}$ \\
\hline$\kappa$ & +1 & -1 & -3 \\
$\mu$ & +1 & 0 & -2 \\
$\tau$ & 0 & 0 & -2 \\
$\eta$ & +1 & 0 & -2 \\
$\chi$ & 0 & -1 & -3 \\
$\omega$ & +1 & - & -2 \\
\hline
\end{tabular}

during the time interval analyzed. Note, also, that in the following day the maximum upward velocity is only $\sim 1 \mathrm{~km} \mathrm{~s}^{-1}$ (see Table 3).

In Fig. 13a we show the maximum downflow velocities (in absolute value) measured in the preceding leg of AFs $\beta$ during the 4 sequences already considered in Fig. 12. In this case we note a significant increase in the measured velocity during the time interval analyzed. The same trend of increasing velocity is found in both the following and the preceding ends of AF $\gamma$ (see Fig. 13b), as well as a systematic greater downflow in the preceding leg.

A similar evaluation is not possible on July 15, because the differences in the los velocities measured among different sequences are within the estimated errors $\left(\sim 1 \mathrm{~km} \mathrm{~s}^{-1}\right)$.

For the other features observed in NOAA 10407, we have deduced that all the pores observed in this region are characterized, at a chromospheric level, by strong $\left(\sim 4 \mathrm{~km} \mathrm{~s}^{-1}\right)$ downflows, that the chromospheric area overlying the sunspot umbra shows an upward motion $\left(v \sim 2 \mathrm{~km} \mathrm{~s}^{-1}\right)$, and that the surrounding network is characterized by both upward and downward motion.

To complete our analysis of the plasma velocities along the line of sight, we used the Fe I line at 5576.012 $\AA$, in order to evaluate their values at the photospheric level. The results obtained, however, do not show any peculiar pattern, because the differences in los velocity noticed among different areas or structures in the examined active region are within the estimated errors $\left(<0.2 \mathrm{~km} \mathrm{~s}^{-1}\right)$.

For the plasma horizontal motions near the photospheric footpoints of the considered arch structures, determined by the cross-correlation procedure adopted in the analysis of the broad band images (see Sect. 3), we did not find any significant systematic motion in the region considered: the pattern appears random, suggesting a turbulent scenario for the rise of the magnetic flux tubes, at least at a photospheric level. This result is analogous to that found in Paper I. 


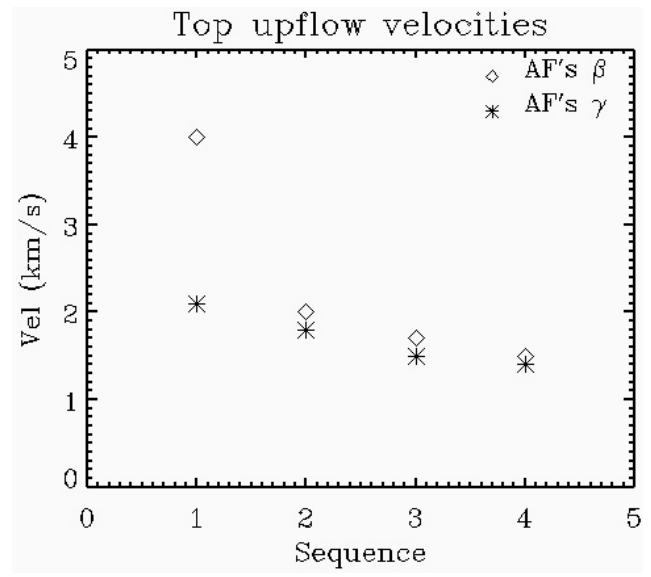

Fig. 12. Plots of the maximum upflow velocities measured at the top of AFs $\beta$ and $\gamma$ during each sequence on July 14.

\section{Discussion}

We have studied the morphology, evolution and dynamics of the magnetic elements in a short-lived active region with a lifetime of 7 days (NOAA 10407). We compare these results with those obtained for the recurrent active region NOAA 10050 studied in Paper I.

The first evidence of this emerging active region is observed in the EIT bands (forming in the transition region and lower corona). In about $7 \mathrm{~h}$ it appears almost contemporaneously in $\mathrm{H} \alpha$ (chromosphere) and white light (photosphere). The case of the recurrent active region NOAA 10050 is slightly different. This region first appeared in EIT images, about $6 \mathrm{~h}$ later in $\mathrm{H} \alpha$, but only after more than $13 \mathrm{~h}$ in white light. This might be related to the larger size of the recurrent region, which implies a longer process of formation. However, the results concerning the time delay between the first appearance of the active region in the lower corona and that in the underlying layers cannot be taken as conclusive, due to the low spatial and temporal resolution of the full disk images used.

The appearance of both examined regions in the EIT bands corresponds to a marked increase in the magnetic flux that continues afterwards: however, the total increase is by about one order of magnitude for the recurrent active region, while for the short-lived one it is by only a factor of two. Moreover, the magnetic flux in NOAA 10050 is generally about two orders of magnitude higher than in NOAA 10407. In addition, we notice the decrease in the magnetic flux and the complete decay of the latter region, while the former one, being recurrent, shows a daily emergence of new magnetic flux during the eight days of observation, up to its setting at the West limb.

As for the displacement of the centre of symmetry of each magnetic polarity, we note a more complex situation in the short-lived region, where only one polarity appears to move away from the magnetic inversion line, while the other is directed toward it (note that the absolute value of the displacement velocity is similar for both polarities). This is noticed also when we consider just the pores where the AFS is rooted, neglecting the single sunspot characterized by a positive magnetic polarity which emerges during the first phases of NOAA 10407

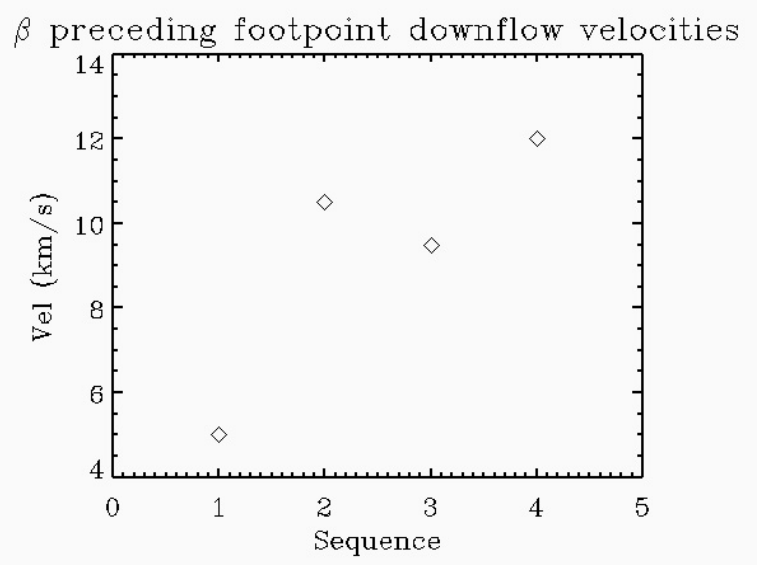

(a)

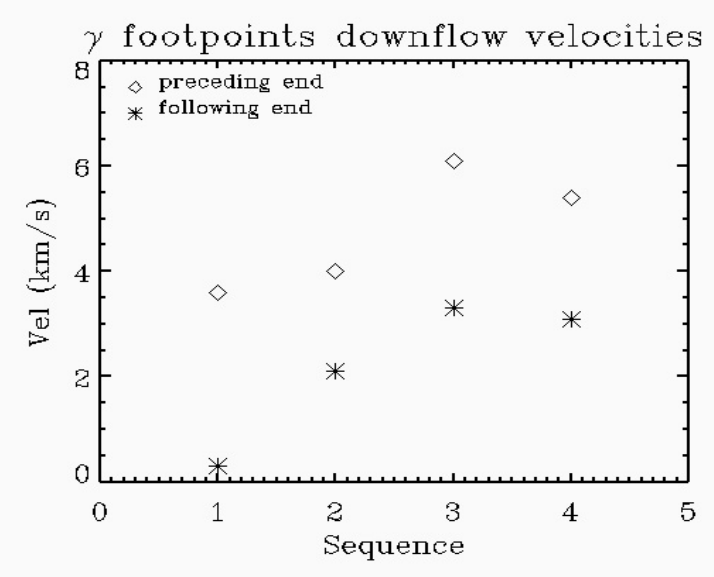

(b)

Fig. 13. Plot of the maximum downflow velocities (in absolute value) measured during the sequences defined in the text in the preceding end of $\mathrm{AF} \beta \mathbf{a}$ ) and in the preceding and following ends of $\mathrm{AF} \gamma \mathbf{b}$ ).

and subsequently appears connected to magnetic elements outside the active region. The peculiar direction of the negative polarity motion does not appear in agreement with the models describing emerging flux tubes (e.g., van Driel \& Petrovay 1990; Petrovay et al. 1990; Howard 1991; Caligari et al. 1995; Moreno-Insertis 1997) and suggests that the flux emergence in this compact region occurs in a more turbulent way with respect to the case of the recurrent active region, where both polarities regularly move away from the magnetic inversion line. Note, however, that the values of the displacement velocity for the two active regions examined do not differ considerably.

The arches of the AFS observed in NOAA 10407 show an upward motion at their tops and generally downward motion at their extremities. The horizontal motion near the photospheric footpoints, conversely, does not show any systematic behaviour. The values of both upflow and downflow velocities measured on July 15 are lower than those obtained on July 14, so confirming the decrease during the evolution of the active region shown in Paper I. The velocities measured on these days, i.e. on the fourth and fifth day of the active region lifetime, are consistent with the velocities obtained on the corresponding days during the emergence and evolution of NOAA 10050. 
A significant increase in the downflow velocities at both footpoints of the arches occurs over about 45 min on July 14 (see Fig. 13). On the other hand, the following day these velocities appear reduced by a factor of about 2 . This aspect should be investigated in more detail during future observations of the emergence and evolution of active regions.

We also note an asymmetry in the downflows, the velocity in the preceding footpoints being systematically higher than that the following ones. This asymmetry, which can be partially determined by geometrical effects (like a greater inclination of the f-leg, see Paper I), decreases from July 14 to 15 , during the days of observations carried out by THEMIS. The correction of the velocities for the line of sight effect is negligible for the heliographic position of the active region on both considered days (see Table 1). Moreover, the lengths of the arch filaments (up to a maximum of $\sim 15000 \mathrm{~km}$ ) imply nearly coincident lines of sight for the preceding and the following footpoints of each arch.

The noticed asymmetry is not in agreement with that discussed in Paper I, where stronger downflow velocities are reported in the following footpoints. On the other hand, it is consistent with the results reported by Cauzzi et al. (1996), who found higher downflows in the p-side polarities of three young active regions.

In both the recurrent and short-lived active region the downflow velocities are higher in the footpoints of the AFS arches characterized by a positive magnetic polarity, even if in the former the magnetic field appears stronger for the negative polarity (see the MDI magnetogram reported in Fig. 8 of Paper I), while the opposite occurs in the latter (see Fig. 6). Taking into account the discussion in Paper I, we can conjecture that the action of the Coriolis force is not able to generate a significant flow along the field lines opposite to the direction of rotation even for upflow velocities of the order of $1 \mathrm{~km} \mathrm{~s}^{-1}$ because the length of the rising flux tube is not sufficient, so that a higher downflow in the following footpoints cannot be detected. Its observation in the preceding footpoints could be due to the residual longitudinal motion in the original mechanical equilibrium, which requires a flow along the field lines compensating the magnetic flux stresses so that the velocity increases with the field strength. The magnetic field appears stronger in the preceding footpoints of the AFS arches detected in NOAA 10407.

Another significant difference between NOAA 10050 and NOAA 10407 is given by the plasma motion measured in the sunspots. While in the former region only downflows with velocities below $2 \mathrm{~km} \mathrm{~s}^{-1}$ are observed, in the latter the sunspot umbra shows an upward motion with $v \sim 2 \mathrm{~km} \mathrm{~s}^{-1}$. The pores exhibit downward motion in both cases.

\section{Conclusions}

The analysis of high resolution images has given us the possibility to highlight some new observational signatures and characteristics of active region formation and evolution.

The results obtained in this analysis indicate that the general behaviour of a short-lived active region during its emergence and evolution has some similarities with those noticed for a region that undergoes complete evolution, but it also has some evident differences.

The common features observed in both long-lived and short-lived active regions are:

- The ARs are initially observed (as a brightness increase) in the outer atmospheric layers (transition region and corona) and later on (i.e. with a time delay of $\sim 6 \div 7 \mathrm{~h}$ ) in the chromosphere;

- The AR appearance in the outer atmospheric layers is synchronous with the sudden increase of magnetic flux in photosphere;

- The arches of the AFS forming in chromosphere are characterized by a decreasing upward motion during the AR's lifetime;

- The plasma downflow motion in the AFS arches decreases with time;

- The AFS plasma velocity is asymmetrically distributed between the two AFS legs.

The differences observed between long-lived and short-lived active regions are:

- While the short-lived AR appearance in the photosphere is almost synchronous with that in the chromosphere, the time delay between the long-lived AR appearance in the chromosphere and in the photosphere is $\sim 8 \mathrm{~h}$ (larger and stronger flux tube bundles meet a higher resistance and the coalescence phase is more complex and much slower);

- The magnetic flux increase during the AR formation is about one order of magnitude in the long-lived AR and only a factor of 2 in the short-lived AR;

- The displacement of the centre of symmetry of each polarity in the short-lived AR is mainly directed westward;

- The higher downflow velocity is measured on the f-side in the long-lived AR and on the p-side in the short-lived AR.

- Opposite motions occur in the sunspots: downflow in the long-lived and upflow in the short-lived AR.

We believe that the items listed above, concerning new observational results, should be included in future theoretical studies on active region emergence and evolution as new observational rules to be considered (see, e.g., Fragos et al. 2004). The time delay between the AR appearance in the higher and lower atmospheric layers deserves further investigation by higher temporal and spatial resolution telescopes. Future instruments, like those foreseen in the Solar B and Solar Dynamics Observatory, could considerably help in verifying our preliminary result.

All these observational signatures fit quite well with a scenario where the short-lived active region is not anchored in the toroidal, subphotospheric magnetic field and is therefore more subject to turbulence than a long-lived, strongly anchored active region. These differences deserve a deeper investigation. 
Acknowledgements. The authors wish to thank E. Catinoto, P. Costa and S. Sciuto for their kind support and cooperation in getting white light and $\mathrm{H} \alpha$ images at the INAF-Catania Astrophysical Observatory. The warm and fruitful help by D. Del Moro and the THEMIS resident staff is acknowledged. The authors also wish to thank the teams of BBSO and TRACE, for participating in the coordinated campaign of observations that allowed this investigation. This work was supported in part by the Italian Ministry of Education, University and Research, in part by the Istituto Nazionale di Astrofisica (INAF) and in part by the Catania University.

\section{References}

Alissandrakis, C. E. 1981, A\&A, 100, 197

Arnaud, J., Mein, P., \& Rayrole, J. 1998, in a Crossroads For European Solar and Heliographic Physics, Tenerife, ESA SP-417, 213

Bruzek, A. 1968, IAUS, 35, 293

Caligari, P., Moreno-Insertis, F., \& Schussler, M. 1995, ApJ, 441, 886

Cauzzi, G., Canfield, R. C., \& Fisher, G. H. 1996, ApJ, 456, 850

Cavallini, F. 1998, A\&A, 128, 589

Contarino, L., Romano, P., Yurchyshyn, V. B., \& Zuccarello, F. 2003, Sol. Phys., 216, 173

Delaboudinière, J.-P., Artzner, G. E., Brunaud, J., et al. 1995, Sol. Phys., 162, 291
Fragos, T., Rantsiou, E., \& Vlahos, L. 2004, A\&A, 420, 719

Handy, B. N., Acton, L. W., Kankelborg, C. C., et al. 1999, Sol. Phys., 187, 229

Howard, R. F. 1991, Sol. Phys., 134, 233

Kitai, R., Funakoshi, Y., Ueno, S., Sano, S., \& Ichimoto, K. 1997, PASJ, 49, 513

Kurokawa, H., \& Kawai, G. 1993, ASP Conf. Ser., 46, 507

Moreno-Insertis, F. 1997, Mem. S.A.It., 68, 429

Petrovay, K., Brown, J. C., van Driel-Gesztelyi, L., et al. 1990, Sol. Phys., 127, 51

Rutten, R. J. 1999, ASP. Conf. Ser., 183, 147

Scherrer, P. H., Bogart, R. S., Bush, R. I., et al. 1995, Sol. Phys., 162, 129

Shibata, K., Tajima, T., \& Steinolfson, R. S. 1989, ApJ, 345, 584

Spadaro, D., Lanzafame, A. C., Consoli, L., et al. 2000, A\&A, 359, 716

Spadaro, D., Billotta, S., Contarino, L., Romano, P., \& Zuccarello, F. 2004, A\&A, 425, 309

van Driel-Gesztelyi, L., \& Petrovay, K. 1990, Sol. Phys., 126, 285

van Driel-Gesztelyi, L. 2002, IAU Coll., 188, ESA SP-505, 113

Yoshimura, K., \& Kurokawa, H. 1999, ApJ, 517, 964

Zuccarello, F., Contarino, L., Romano, P., et al. 2002, INAF-OACt Internal report N. 1

Zwaan, C. 1985, Sol. Phys., 100, 397 